\title{
Hartmann wavefront measurements at FLASH
}

Barbara Keitel, Bernhard Flöter, Svea Kreis, Marion Kuhlmann, Klaus Mann, et al.

Barbara Keitel, Bernhard Flöter, Svea Kreis, Marion Kuhlmann, Klaus Mann, Tobias Mey, Elke Plönjes, Bernd Schäfer, Kai Tiedtke, "Hartmann wavefront measurements at FLASH," Proc. SPIE 8778, Advances in X-ray Free-Electron Lasers II: Instrumentation, 877814 (3 May 2013); doi: $10.1117 / 12.2020850$

SPIE. Event: SPIE Optics + Optoelectronics, 2013, Prague, Czech Republic 


\title{
Hartmann wavefront measurements at FLASH
}

\author{
Barbara Keitel*º $^{\mathrm{b}}$, Bernhard Flöter ${ }^{\mathrm{a}}$, Svea Kreis ${ }^{\mathrm{b}}$, Marion Kuhlmann ${ }^{\mathrm{b}}$, Klaus Mannª ${ }^{\mathrm{a}}$, Tobias Mey ${ }^{\mathrm{a}}$, \\ Elke Plönjes ${ }^{\mathrm{a}}$, Bernd Schäfer ${ }^{\mathrm{a}}$, Kai Tiedtke ${ }^{\mathrm{b}}$ \\ ${ }^{a}$ Laser-Laboratorium Göttingen e.V., Hans-Adolf-Krebs-Weg 1, 37077 Göttingen, Germany; \\ ${ }^{\mathrm{b}}$ Deutsches Elektronen-Synchrotron DESY, Notkestrasse 85, 22607 Hamburg, Germany;
}

\begin{abstract}
We report on online measurements of photon beam parameters during mirror alignment in the soft $\mathrm{x}$-ray spectral region of FLASH, the free-electron laser in Hamburg. A compact Hartmann sensor operating in the wavelength range from 6 to $35 \mathrm{~nm}$ was used to determine the wavefront quality of individual free-electron laser (FEL) pulses during the alignment procedure as well as aberrations. Beam characterization and alignment of beamline BL3 was performed with $\lambda_{13.5 \mathrm{~nm}} /$ 116 accuracy for wavefront rms $\left(w_{r m s}\right)$. Second moment beam parameters are computed using a spherical reference wavefront generated by a $5 \mu \mathrm{m}$ pinhole. The Hartmann sensor was used for alignment of the ellipsoidal focusing mirror of beamline BL3, resulting in a reduction of $w_{r m s}$ by $33 \%$.
\end{abstract}

Keywords: Wavefront, Free-electron laser, Hartmann sensor, soft x-ray

\section{INTRODUCTION}

FLASH, the free-electron Laser in Hamburg is operating in the soft x-ray spectral region based on the self-amplified spontaneous emission (SASE) process in which laser emission is build up from spontaneous undulator radiation. Due to the stochastic nature of this process the beam characteristics relevant for user experiments differ from pulse to pulse, leading to a strong requirement of single pulse photon diagnostic and online characterization of the parameters. ${ }^{1,2}$

Hartmann and Hartmann-Shack sensors are used for real-time wavefront detection and beam characterization in the near infrared, visible and soft x-ray spectral region. Both, the directional (wavefront) and intensity (beam profile) distribution of the radiation field are provided for single pulses. This enables the evaluation of paraxial beam parameters such as beam width $d$, divergence $\theta$, beam propagation factor $M^{2}$, Rayleigh length $z_{R}$, waist position $z_{0}$ and waist diameter $d_{0}{ }^{3}$ The prediction of the intensity profile in any plane is found by solving the Fresnel-Kirchhoff integral. ${ }^{4}$ In this paper, we present the results from measurements at the FLASH beamline BL3 performed with a compact Hartmann sensor jointly developed by Laser-Laboratorium Göttingen (LLG) and DESY for photon diagnostics, beamline alignment and monitoring of FEL radiation at FLASH ${ }^{5,6}$. The determination of beam parameters relevant for many user experiments as well as the alignment of the ellipsoidal focusing mirror is described.

\section{OPERATION PRINCIPLE}

\subsection{The Hartmann wavefront sensor}

The setup of our Hartmann sensor (see figure 1) is based on the description of Hartmann ${ }^{7}$ and consists of a pinhole array and a 14 bit camera with a charge-coupled device (CCD) chip in a distance $l=198,251 \mathrm{~mm}$ behind the pinhole array. The distance represents a compromise between attainable wavefront sensitivity and spatial resolution given by the pitch of the pinhole array. The CCD chip has a size of $8.9 \mathrm{~mm}$ x $6.7 \mathrm{~mm}$ with $6.45 \mu \mathrm{m}$ x $6.45 \mu \mathrm{m}$ pixel size and is coated with a phosphor $\left(\mathrm{Gd}_{2} \mathrm{O}_{2} \mathrm{~S}: \mathrm{Tb}\right.$, grain size $1-2 \mu \mathrm{m}$, central emission wavelength $\left.545 \mathrm{~nm}\right)$ for converting the soft $\mathrm{x}$-rays into visible light. The Hartmann plate, a pinhole array, made of a $20 \mu \mathrm{m}$ thick nickel foil with electroformed holes $(75 \mu \mathrm{m}$ diameter and $250 \mu \mathrm{m}$ pitch) by Precision Eforming divides the incoming beam into an array of smaller beams. The position and intensity of the beams coming from each sub-aperture is measured with the CCD camera.

*barbara.keitel@desy.de; phone +49 40 8998-4245; fax +49 40 8994-4245;

http://photon-science.desy.de/facilities/flash/index_eng.html

Advances in X-ray Free-Electron Lasers II: Instrumentation, edited by

Thomas Tschentscher, Kai Tiedtke, Proc. of SPIE Vol. 8778, 877814 - (c) 2013

SPIE - CCC code: 0277-786X/13/\$18 - doi: 10.1117/12.2020850 

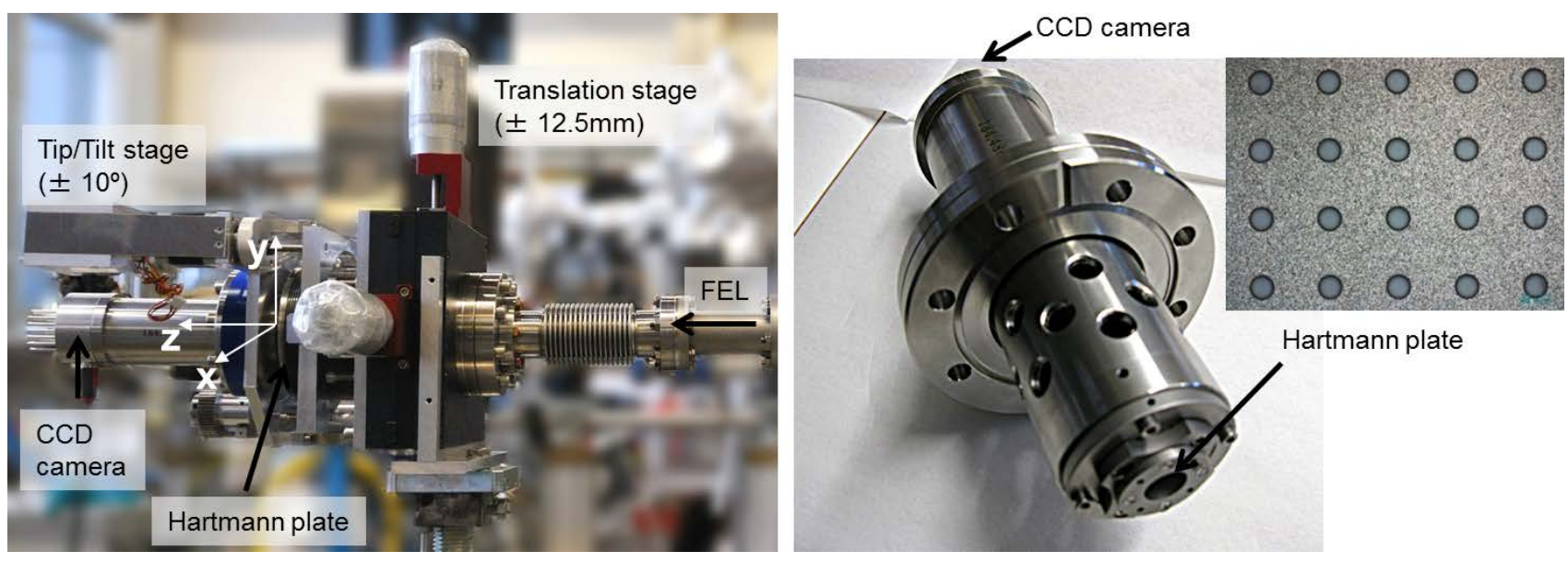

Figure 1. left: Hartmann sensor, right: Inner part of the Hartmann sensor with the Hartmann plate holder. In the inset a light optical microscopic image (magnification 10x) of part of the Hartmann plate with electroformed holes is shown.

The displacement of a spot centroid $\Delta x$ divided by the distance $l$ yields the local wavefront gradient inside one subaperture relative to a known reference wavefront (see figure 2). The wavefront is reconstructed from those local gradients in a modal approach according to the references ${ }^{8,9,10}$, using 37 Zernike polynomials in the notation of reference $^{11}$. Summation over all pixel values belonging to the individual sub-apertures samples the intensity distribution or beam profile $I(x, y)$. The Hartmann data, consisting of sampled intensity and wavefront gradients $\beta_{x}$, facilitate to compute the first and second order moments of the spatial $(x, y)$ and angular $(u, v)$ coordinates over the intensity distribution ${ }^{3}$.

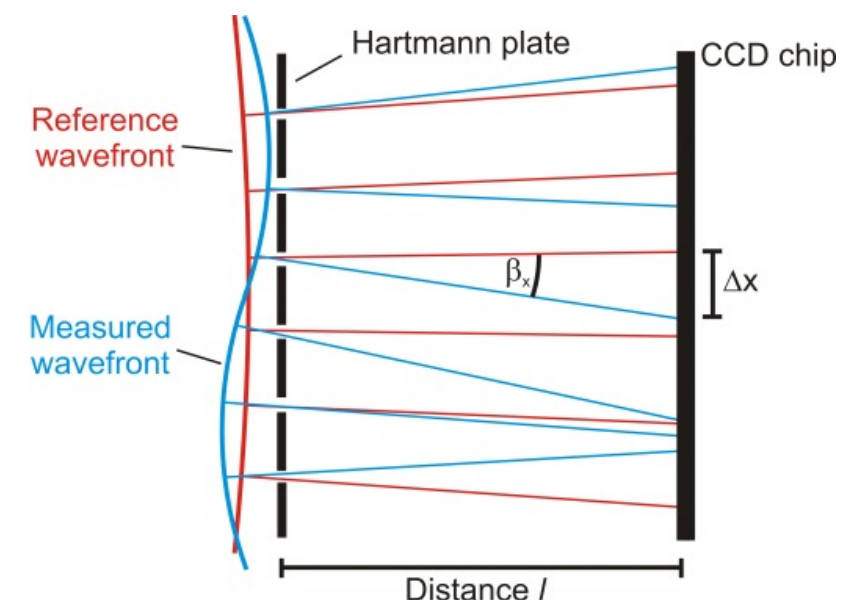

Figure 2. Hartmann principle: The incoming beam (blue) is divided into an array of partial beams by the Hartmann plate. The centroid deviation $\Delta x$ from the known reference spot position divided by the distance $l$ yields the local wavefront gradient $\beta_{x}$ relative to the reference wavefront (red).

This information is sufficient to compute the beam parameters ${ }^{3,11}$ as beam width $d$, divergence $\theta$, beam propagation factor $M^{2}$, waist diameter $d_{0}$, Rayleigh length $z_{R}$ and waist position $z_{0}$ of paraxial coherent beams. Influences from partial coherence are neglected in this evaluation. The computation of second moment beam parameters from Hartmann data for soft x-ray radiation and the general agreement with caustic scan techniques was reported in reference ${ }^{5}$. Once 
intensity and phase of the beam are known from the Hartmann measurements, the Fresnel-Kirchhoff propagation yields the intensity distribution at different positions $\mathrm{z}^{4}$

The area of interest (AOI) for wavefront and beam profile evaluation is selected by clipping noise at a level of $1 \%$ of the full dynamic range of the CCD camera. The evaluation radius $a$ is defined by the largest circle inscribed into this AOI. The quality of the wavefront is expressed in terms of wavefront peak-to-valley $w_{P V}$ and wavefront root-mean-square $w_{r m s}$ values. Both are calculated after subtraction of tilt and the best-fitting-square.

\subsection{Reference wavefront}

For wavefront evaluation the Hartmann sensor requires a reference wavefront. Therefore, a $5 \mu \mathrm{m}$ pinhole was placed into the FEL beam to generate a spherical wavefront ${ }^{12}$. The pinhole was located $110 \mathrm{~mm}$ upstream of the focal plane at beamline BL2 (ellipsoidal mirror with $2 \mathrm{~m}$ focal length) and 3592mm in front of the Hartmann plate. FEL pulse train of 30 bunches at a wavelength of $13.5 \mathrm{~nm}$ were used for calibration measurements. The single pulse energy of about $80 \mu \mathrm{J}$ was attenuated using a meshless, 216nm thick Si filter foil to prevent destruction of the pinhole by the FLASH photon beam. For improved signal-to-noise ratio, 100 camera frames, each containing a single pulse train, were averaged to create the reference shown in figure 3 . The beam profile was centered on the wavefront sensor with the optical axis perpendicular to the CCD chip. A slightly bigger pinhole (see right bottom in figure 3) in the Hartmann plate defines the sensor orientation to the beam and makes it easier to identify individual sub-apertures.

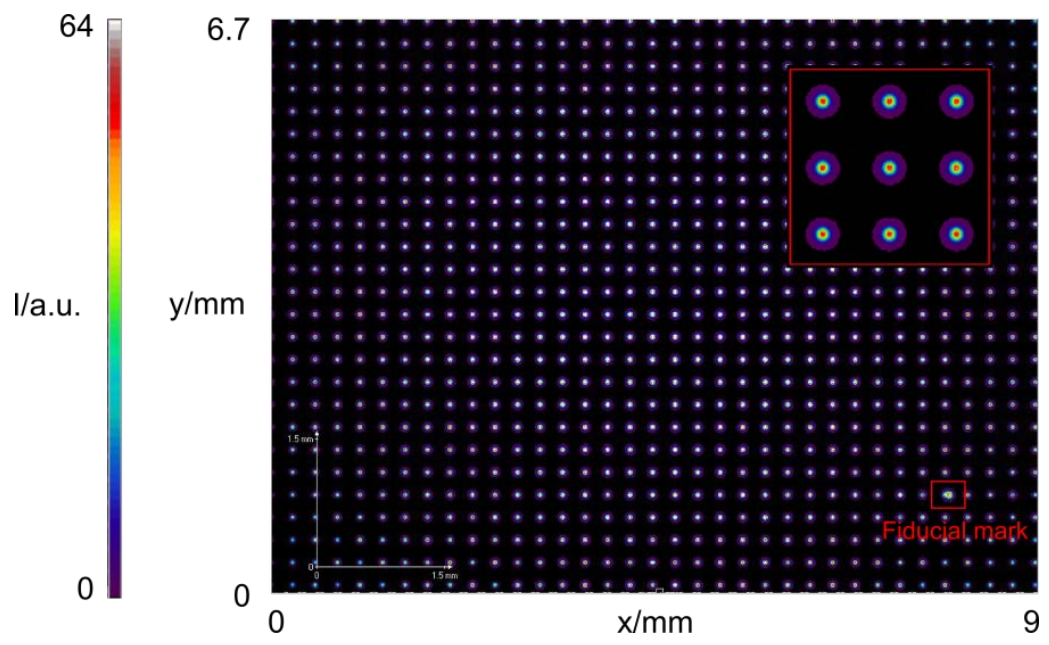

Figure 3. A reference spot pattern is created by diffraction from a $5 \mu \mathrm{m}$ pinhole. For alignment purposes, one pinhole (Fiducial mark) bottom right on the Hartmann plate shows a distinct size and shape. It defines the sensor orientation and helps identifying individual apertures.

The average relative single pulse repeatability of the wavefront measurements was determined against the reference shown in figure 3 . Therefor a series of 100 single FEL pulses ( 1 bunch per train) was recorded behind the $5 \mu \mathrm{m}$ pinhole at $\lambda=13.5 \mathrm{~nm}$. Using an evaluation radius of $a=3.07 \mathrm{~mm}$, a mean deviation for the peak-to-valley value $w_{P V}$ of $\lambda / 19$ and for the root-mean-square value $w_{r m s}$ of $\lambda / 116$ was calculated. Tip/Tilt and defocus are subtracted prior to the computation of the $w_{P V}$ and $w_{r m s}$ values.

\section{BEAMLINE COMMISSIONING AT BL3}

The Hartmann sensor was placed about 850mm downstream of the expected focal plane at FLASH beamline BL3 (see figure 4) to adapt the beam size to the detector area. The measurements were performed in January 2011 using a 
wavelength of $13.3 \mathrm{~nm}$ in single bunch mode with $0.5 \mathrm{nC}$. Two $10 \mathrm{~mm}$ apertures in an upstream distance of about $48.3 \mathrm{~m}$ and $52.8 \mathrm{~m}$ from the focusing ellipsoidal mirror (focal length $2 \mathrm{~m}, 3^{\circ}$ grazing incidence angle, amorphous carbon coating) were used. The intensity of about $65 \mu \mathrm{J}$ was attenuated to about $2 \mu \mathrm{J}$ per pulse using $0.01 \mathrm{mbar}$ Xe in the gas attenuator; the use of transmission filters for further attenuation was not needed. The camera of the wavefront sensor was triggered by the fast shutter which allows us to measure at $10 \mathrm{~Hz}$ observing online the changes of the Zernike coefficients, $w_{r m s}$, $w_{P V}$ and further beam parameters during the mirror movement.

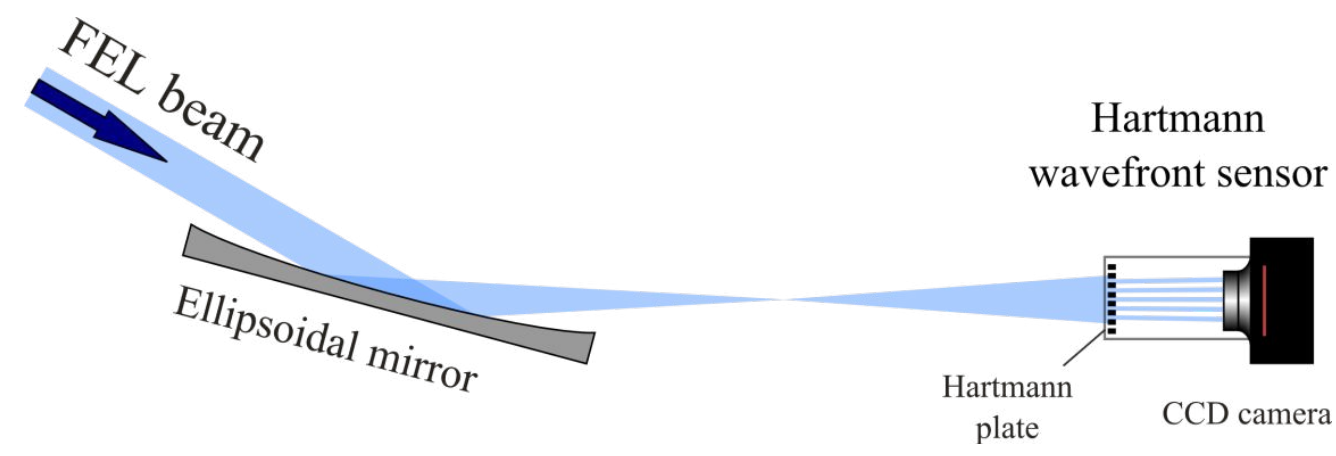

Figure 4. Setup used for the online adjustment of the ellipsoidal grazing incidence focusing mirror of beamline BL3 at FLASH. The ellipsoidal mirror is located in a distance of $72.2 \mathrm{~m}$ to the source.

The newly installed focusing mirror of beamline BL3 was aligned by successively moving the mirror with respect to the yaw and pitch angles around the pre-aligned position. The goal of alignment procedure with the wavefront sensor is to maximize the Strehl value, which is, according to the Maréchal formula Strehl $\approx 1-\left(\frac{2 \pi}{\lambda} w_{r m s}\right)^{2}$, equivalent to minimizing $w_{r m s}$, with $w_{r m s}$ given in $\mathrm{nm}^{4,13}$.

First the yaw angle was aligned such that the astigmatism is oriented along the vertical and horizontal coordinate axis. Therefore, starting at a mirror yaw angle of $0 \mathrm{mrad}$, we scanned in a range between $-0.5 \mathrm{mrad}$ and $1.5 \mathrm{mrad}$. Afterwards, the pitch angle was adjusted in a rage of $0.43 \mathrm{mrad}$ and $1.43 \mathrm{mrad}$ to minimize the $w_{r m s}$ value. For a yaw angle of $0.65 \mathrm{mrad}$ we again optimized the pitch angle in a fine-adjustment, finally leading to a reduction of the $w_{r m s}$ value by $68 \%$ from $4 \mathrm{~nm}$ to $1.3 \mathrm{~nm}$ and of $w_{P V}$ by $56 \%$ from $27 \mathrm{~nm}$ to $12 \mathrm{~nm}$. In parallel to the mirror movement the astigmatism Zernike coefficients were calculated online to retain information on the success of alignment. 

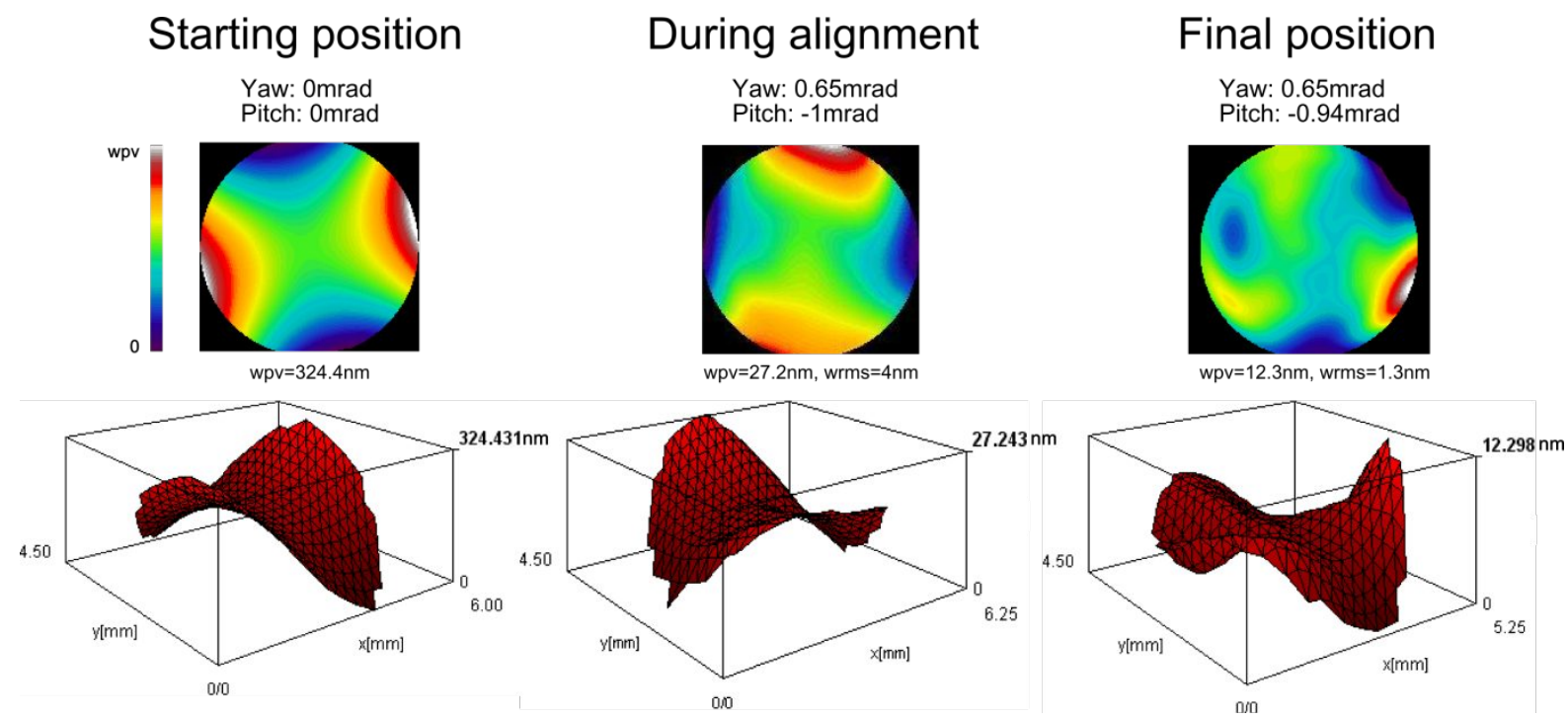

Figure 5. Wavefronts measured at different steps of the alignment procedure of the ellipsoidal focusing mirror at FLASH beamline BL3. The measurements are performed at a wavelength of $13.3 \mathrm{~nm}$, in single bunch mode and use of the gas attenuator. The gas attenuator was filled with $0.01 \mathrm{mbar}$ Xe to decrease the pulse energy to about $2 \mu \mathrm{J}$ per pulse.

In figure 5 wavefronts (evaluation radius $\mathrm{a}=2.38 \mathrm{~mm}$ ) at starting conditions as well as during the alignment procedure and for the final mirror position are shown. The wavefronts shown for the starting conditions and during the alignment are single pulse measurements whereas the wavefront for the final alignment shows an average of 100 single frames on the CCD chip. In table 1 the evaluated beam parameters and astigmatism Zernike aberrations are summarized. The Strehl ratio and the focal spot quality are improved accordingly (see table 1). From the determined final waist position we calculated the focus position to be in a distance of $528.7 \mathrm{~mm}$ from the flange of the last beamline valve.

Table 1. Beam parameter and Zernike coefficients for different steps of alignment. For the starting position and during the alignment single pulse parameters are given. For the final mirror position parameters are calculated for an average of 100 single pulses.

\begin{tabular}{|c|c|c|c|c|c|c|}
\hline & \multicolumn{2}{|c|}{$\begin{array}{l}\text { Second moment beam } \\
\text { parameters at starting } \\
\text { position }\end{array}$} & \multicolumn{2}{|c|}{$\begin{array}{l}\text { Second moment beam } \\
\text { parameters during } \\
\text { alignment }\end{array}$} & \multicolumn{2}{|c|}{$\begin{array}{l}\text { Second moment beam } \\
\text { parameters at final } \\
\text { position }\end{array}$} \\
\hline & $\mathrm{x}$ & $\mathrm{y}$ & $x$ & $\mathrm{y}$ & $x$ & $\mathrm{y}$ \\
\hline$w_{P V}[\mathrm{~nm}]$ & \multicolumn{2}{|c|}{324.43} & \multicolumn{2}{|c|}{27.24} & \multicolumn{2}{|c|}{12.19} \\
\hline$w_{r m s}[\mathrm{~nm}]$ & \multicolumn{2}{|c|}{-} & \multicolumn{2}{|c|}{3.97} & \multicolumn{2}{|c|}{1.31} \\
\hline Beam propagation factor $M^{2}$ & \multicolumn{2}{|c|}{-} & \multicolumn{2}{|c|}{1.94} & \multicolumn{2}{|c|}{1.82} \\
\hline Beam propagation factor $M_{i}^{2}$ & - & - & 2.25 & 1.97 & 1.99 & 1.83 \\
\hline Beam diameter $d[\mathrm{~mm}]$ & 3.64 & 3.97 & 3.86 & 4.06 & 3.34 & 3.85 \\
\hline Waist position $z_{0}[\mathrm{~mm}]$ & -882.85 & -813.87 & -846.63 & -852.61 & -848.94 & -848.63 \\
\hline Waist diameter $d_{0}[\mu \mathrm{m}]$ & 107.98 & 90.37 & 8.8 & 7.4 & 9.1 & 7.2 \\
\hline Astigmatism $Y^{2}-X^{2}[\mu \mathrm{m}]$ & \multicolumn{2}{|c|}{-0.1338} & \multicolumn{2}{|c|}{0.0116} & \multicolumn{2}{|c|}{-0.00113} \\
\hline Astigmatism $X Y[\mu \mathrm{m}]$ & \multicolumn{2}{|c|}{0.8632} & \multicolumn{2}{|c|}{0.0034} & \multicolumn{2}{|c|}{-0.00145} \\
\hline
\end{tabular}

The Hartmann data recorded before and after the alignment procedure were used to compute beam profiles close to the waist of the FEL beam by numerical propagation via Kirchhoff-Fresnel integral ${ }^{5}$. The corresponding simulated beam profiles are displayed in figure 6. Before the alignment (upper profiles) the strong wavefront astigmatism leads to a 
change of the profile orientation before and after the focus, whereas after the alignment a circular beam profile in all planes is observed (lower profiles).

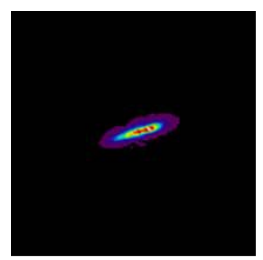

$-50 \mathrm{~mm}$

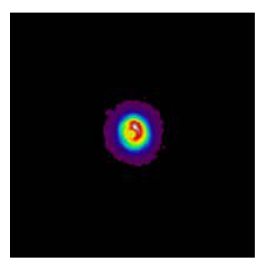

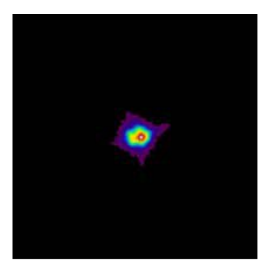

Focal plane

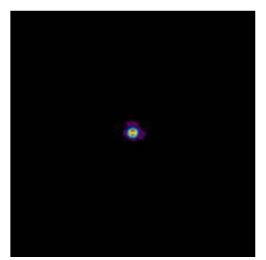

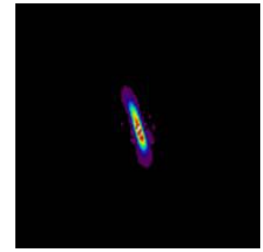

$+50 \mathrm{~mm}$

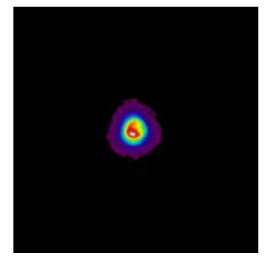

Before alignment

After alignment

Figure 6. Beam profiles computed for the near-waist region by numerical propagation of Hartmann data before (above) and after (below) the alignment procedure.

\section{CONCLUSION}

In this paper we report on beam characterization measurements for the free-electron laser FLASH using a Hartmann wavefront sensor for the soft x-ray spectral region with high stability and accuracy. The compact device is mounted on a CF63 vacuum flange and can easily be attached to different beamlines or experimental chambers. This Hartmann sensor with high accuracy is now available for FLASH users and can be applied for evaluation of wavefront, Zernike coefficients, intensity profiles and different beam parameter. A single pulse wavefront repeatability was determined against a spherical reference wavefront, corresponding to $\lambda / 116$ at $\lambda=13.5 \mathrm{~nm}$. We described an alignment procedure of the ellipsoidal focusing mirror of FLASH beamline BL3. The astigmatic distortion dominating the wavefront before the adjustment could strongly $(>60 \%)$ be reduced to $\lambda / 10\left(w_{r m s}\right)$ at $13.3 \mathrm{~nm}$.

\section{ACKNOWLEDGEMENTS}

Support of the FLASH user facility, in particular the funding of wavefront sensors through the BMBF program FSP301FLASH, is greatly acknowledged. This work is partly supported by 'IRUVX-PP', EU co-funded project under FP7 (Grant Agreement 211285). We also acknowledge the support from Deutsche Forschungsgemeinschaft within SFB755 'Nanoscale Photonic Imaging'.

\section{REFERENCES}

[1] Tiedtke, K. et al., "The soft x-ray free-electron laser FLASH at DESY: beamlines, diagnostics and endstations”, New J. of Phys. 11, 023029 (2009).

[2] Kuhlmann, M. et al., "Wave-front observations at FLASH", Proceedings of FEL 2006, X-ray Optics and Detectors, 794-797 (2006).

[3] Schäfer, B. and Mann, K., "Determination of beam parameters and coherence properties of laser radiation by use of an extended Hartmann-Shack wave-front sensor”, Appl. Opt. 41(15), 2809-2817 (2002).

[4] Born, M. and Wolf, E., [Principles of Optics], $6^{\text {th }}$ ed., Cambridge University Press, Cambridge, (1985).

[5] Flöter, B. et al., "EUV Hartmann sensor for wavefront measurements at the Free-electron Laser in Hamburg", New J. of Phys. 12, 083015 (2010). 
[6] Flöter, B. et al., "Beam parameters of FLASH beamline BL1 from Hartmann wavefront measurements”, Nucl. Instr. And Meth. A 635, 108-112 (2011).

[7] Hartmann, J., “ Bemerkungen über den Bau und die Justierung von Spektrographen”, Z. Instrumentenkd. 20, 47-58 (1900).

[8] Cubalchini, R., "Modal wave-front estimation from phase derivative measurements”, J. Opt. Soc. Am. 69(7), 972-977 (1979).

[9] Noll, J. R., "Phase estimates from slope-type wave-front sensors", J. Opt. Soc. Am. 68(1), 139-140 (1978).

[10] ISO 15367, "Lasers and laser-related equipment - Test methods for determination of the shape of a laser beam wavefront”, (2005).

[11] Schäfer, B. et al., "Hartmann-Shack wave front measurements for real time determination of laser beam propagation parameters”, Rev. Sci. Instrum. 77, 053103 (2006).

[12] Mercère, P. et al., "Hartmann wave-front measurement at $13.4 \mathrm{~nm}$ with $\lambda_{\text {EUV }} / 120$ accuracy”, Opt. Lett. 28 (17), 1534-1536 (2003).

[13] Maréchal, A.,” Étude des effects combines de la diffraction et des aberrations géométriques sur l'image d'un point lumineux", Rev. Opt. 26, 257 (1947). 